\title{
Nutrition Shared Resource
}

National Cancer Institute

\section{Source}

National Cancer Institute. Nutrition Shared Resource. NCI Thesaurus. Code C39480.

The Nutrition Shared Resource provides Cancer Center investigators with support in dietary and nutritional assessment and monitoring, including quantitative measurements of biochemical indicators of diet and other biomarkers in blood and other biological samples, for research that address nutritional or dietary factors in cancer prevention and treatment. The resource provides counseling service for promoting dietary change, surveys, and meta-analyses of available data. 